\title{
Waveguiding in Vertical Cavity Quantum-Well Structure Defined by Ion Implantation
}

\author{
Alex T. H. Li, Member, IEEE, Chun-Chung Chan, and E. Herbert Li, Senior Member, IEEE
}

\begin{abstract}
A theoretical model is presented for investigating AlGaAs/GaAs quantum-well (QW) vertical cavity waveguides defined by impurity-induced disordering. This model is based on a two-dimensional (2-D) description of the implantation profile to produce quantum-well intermixing. The modal propagation constant, power, and field evolution along the nonuniform circular waveguide is analyzed in terms of the coupled-mode equation. The influence of varying the mask dimension and length of cavity on the fundamental mode operation is studied. In a long cavity, guided mode can be supported by using higher implantation energy, however, a larger mask diameter should be used to maintain strong guiding. Result shows that optical lateral confinement is accomplished, with more than $70 \%$ of the power gathered in the cavity. The fraction of power confined in the waveguide is shown to improve by $30 \%$ after annealing. In addition, waveguide loss is estimated to be less than $40 \mathrm{~cm}^{-1}$ which results in less than $1 \mathrm{~dB}$ for our structure.
\end{abstract}

Index Terms-Diffusion process, ion implantation, optical waveguide, propagation, quantum-well devices, quantumwell interdiffusion, quantum-well intermixing, vertical cavity waveguide.

\section{INTRODUCTION}

$\mathbf{I}$ $\mathrm{N}$ the past few years, vertical cavity devices using semiconductor multiple quantum-wells (MQW's) have given rise to a variety of novel devices, such as lasers [1], lightemitting diodes [2], modulators [3], and photodetectors [4]. The use of vertical cavity is of great interest because of their high packing density [5], low-threshold current [6], suitable for parallel free-space optical interconnect [7], and most recently, the blue-green light source for high density data storage [8].

The technique of impurity-induced disordering (IID) of the QW composition between layers has recently been successfully adopted for carrier confinement in the vertical cavity surface emitting laser (VCSEL) [9]. IID has conventionally been used for tailoring the band-gap wavelength and optical properties of MQW's through intermixing of the alloy composition in the hetero-layers for the realization of a tunable and high performance device [10]. Zn impurity-induced QW intermixing allows a precise lateral modification of the optoelectronic properties of semiconductor $\mathrm{QW}$ structures in a controllable and reproducible manner by using simple masking and high temperature $\left(850{ }^{\circ} \mathrm{C}\right)$ diffusion processed on a planar substrate. The technique of ion implantation can serve as a mean of accurate control to introduce the impurities in the region where interdiffusion takes place. The damage

\footnotetext{
Manuscript received July 31, 1997; revised February 23, 1998. This work was supported by the Hong Kong Research Grant Council.

The authors are with the Department of Electrical and Electronic Engineering, University of Hong Kong, Hong Kong.

Publisher Item Identifier S 0733-8724(98)05647-3.
}

introduced by ion-implantation can be recovered by thermal annealing. The development of detailed modeling for the IID defined vertical cavity waveguide is essential for the understanding and ease in optimal design of this type of devices. Although experiments have been performed on IID defined vertically cavity for some time [11], studies on the waveguiding properties are still lacking.

We investigate a vertical cavity structure that utilizes ionimplantation to intermix the lateral circular boundary of the $\mathrm{QW}$ region to achieve lateral optical confinement. A three-dimensional vertical cavity model is developed for AlGaAs/GaAs MQW's. The three-dimensional (3-D) waveguide structure is converted to a two-dimensional (2-D) problem based on the azithumal symmetry. The inherent nonuniformity of the IID waveguide is analyzed with the method of multilayer approximation [12]. The waveguide characteristics such as propagation constant and power distribution for different waveguide dimensions, annealing time, implantation energy and operating wavelength are analyzed. The waveguide structure is assumed to be transitionally invariant along the propagation direction. Optical properties, except phase, are thus considered to be invariant along the waveguide. Variation of the optical properties due to the nonuniformity is accounted for and the algorithm of coupled mode theory is adopted to determine the behavior of the traveling wave. This paper is organized as follows: in Section II, we present a selfconsistent model of vertical cavity waveguide with the effect of implanted impurities, annealing and a 2-D wavelength dependent refractive index profile taken into consideration. In addition, the longitudinal and radial distributions of the optical field are also considered. The results obtained are then combined to determine the characteristics of the waveguide properties, and which are presented in Section III. Conclusion is presented in the last section.

\section{MODEL}

The IID technique provides an efficient way to realize waveguiding structure in photonic integrated circuit [13]. The masked implantation process produces a modification of the QW structure which in turn modifies its refractive index. This creates a refractive index step between the implanted and nonimplanted regions and produces optical lateral confinement. The present work is to develop a model for a 2-D waveguiding structure perpendicular to the QW layers.

The structure to be modeled composes of layers of $\mathrm{Al}$ GaAs/GaAs QW on a thick $\mathrm{Al}_{0.3} \mathrm{Ga}_{0.7} \mathrm{As}$ buffer layer. The schematic of the structure is shown in Fig. 1. In this model, we use masked implantation to selectively inject impurities in 


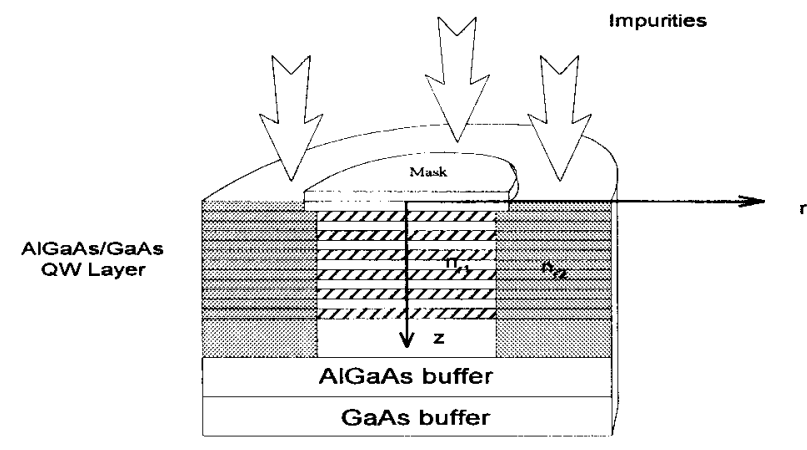

Fig. 1. Schematic of the waveguide structure.

the outer perimeter to enhance the interdiffusion rate there. After annealing (e.g., rapid thermal annealing), the QW's in the implanted region will intermix to a greater extent and thus will have a larger band-gap and thus a lower refractive index, so that a refractive index step is produced to create lateral optical confinement [14]. Therefore a vertical circular waveguide is formed. It should be noted that the refractive index of the waveguide structure fabricated by such technique is inhomogeneous, see Fig. 2.

A mask of radius $a$ is put on top of the as-grown structure, as shown in Fig. 1. Ion implantation is used to inject the impurity into the structure. Based on [15] and with the assumption of circular symmetry, the distribution of ions implanted $F(r, \phi, z)$, where $r$ represents the lateral direction, $\phi$ represents the circular direction and $z$ represents the longitudinal direction and growth axis, through a circular mask of radius $a$ can be expressed by

$$
\begin{aligned}
& F(r, z)=\frac{N_{0}}{\sqrt{2 \pi}\left\langle\Delta R_{p}\right\rangle\left\langle\Delta R_{p l}\right\rangle} \exp \left\{-\frac{\left(z-\left\langle R_{p}\right\rangle\right)^{2}}{2\left\langle\Delta R_{p}\right\rangle^{2}}\right\} \\
& \times\left\{-\frac{\left\langle\Delta R_{p l}\right\rangle}{2}\left[\exp \left(-\left(\frac{b-r}{\left\langle\Delta R_{p l}\right\rangle}\right)^{2}\right)\right.\right. \\
& \left.-\exp \left(-\left(\frac{a-r}{\left\langle\Delta R_{p l}\right\rangle}\right)^{2}\right)\right] \\
& \left.+\frac{\sqrt{\pi} r}{2}\left[\operatorname{erf}\left(\frac{b-r}{\left\langle\Delta R_{p l}\right\rangle}\right)-\operatorname{erf}\left(\frac{a-r}{\left\langle\Delta R_{p l}\right\rangle}\right)\right]\right\}
\end{aligned}
$$

where $\operatorname{erf}(\cdot)$ is the error function; $N_{\circ}$ is the implantation dose of $\mathrm{Ga}^{+}$ion; $b$ is the radius of the waveguide (including core and cladder), see Fig. 1; $R_{p}$ is the projected range, and $\Delta R_{p l}, \Delta R_{p}$ are lateral radial and vertical standard deviations respectively (note that all these three values are governed by implanted ions, implantation energy and substrate material), and $\langle\cdots\rangle$ denotes the expectation value. $F$ is independent of $\phi$ due to symmetry.

After ion-implantation, the structure will be allowed to heat under high temperature (generally $850^{\circ} \mathrm{C}$ ). While annealing, the impurities with concentration $D_{\text {imp }}$ will diffuse in all directions. The instantaneous impurity profile $N(r, z, t)$ at any annealing time $(t)$ can be found by solving the heat equation, which is given below, subject to the initial condition

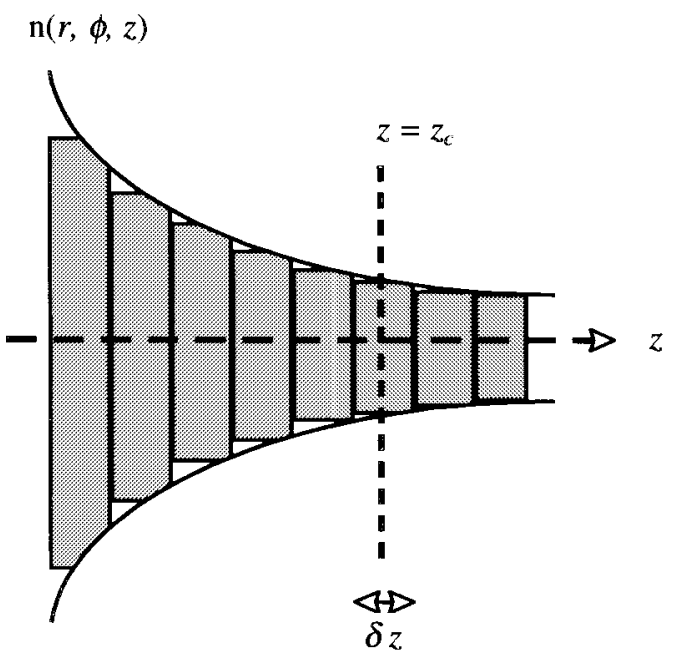

Fig. 2. The approximation scheme for a nonuniform circular waveguide core with a varying refractive-index profile along its propagation direction ( $z$-axis). A series of sections are shown, where $z_{c}$ denotes the centre of each section and $\delta z_{c}$ denotes its width.

$$
\begin{aligned}
& N(r, z, t=0)=F(r, z) \\
& \frac{\partial N(r, z, t)}{\partial t}=D_{\mathrm{imp}} \nabla^{2} N(r, z, t) .
\end{aligned}
$$

The impurity diffusion coefficient $\left(D_{\mathrm{imp}}\right)$ is assumed to be constant during the annealing process, hence, the diffusion equation is linear. By a classical diffusion law, the diffusion coefficient of $\mathrm{Al}$ is proportional to the local impurity density, the corresponding interdiffusion rate can be described as

$$
D_{A l}=\alpha D_{\mathrm{imp}} N(r, z, t)
$$

where $\alpha$ is a constant factor. As a result, we can compute the concentration profile of the $\mathrm{Al}$ by

$$
\frac{\partial C(r, z, t)}{\partial t}=\vec{\nabla} \cdot\left[D_{A l}(r, z, t) \vec{\nabla} C(r, z, t)\right] .
$$

As a result of the circular invariance of the impurity distribution, this equation becomes

$$
\begin{aligned}
\frac{\partial C(r, z, t)}{\partial t}= & D_{A l}\left(\frac{\partial^{2} C}{\partial r^{2}}+\frac{\partial^{2} C}{\partial z^{2}}\right) \\
& +\left(\frac{\partial D_{A l} \partial C}{\partial r^{2}}+\frac{\partial D_{A l} \partial C}{\partial z^{2}}\right)+\frac{D_{A l}}{r} \frac{\partial C}{\partial r} .
\end{aligned}
$$

Once this is solved, we can then match the impurities and hence vacancy profiles with a specific diffusion length $\left(L_{d}\right)$ through a QW interdiffusion calculation [16]

$$
w(z)=w_{o}\left(1-\frac{1}{2}\left[\operatorname{erf}\left(\frac{L_{z}+2 z}{4 L_{d}}\right)\right]+\operatorname{erf}\left(\frac{L_{z}-2 z}{4 L_{d}}\right)\right)
$$

where $w$ is the $A l$ concentration profile of a $\mathrm{QW}$ structure with well width $L_{z}$ and $w_{o}$ is the initial $A l$ concentration. The diffusion length is defined here as $L_{d}=\sqrt{D_{A l}}$ and which represents the extent of interdiffusion. Once we obtained the matched $L_{d}$, the refractive index can be obtained [17]. A 2-D refractive index profile $n(r, z)$ can be constructed by 
calculating all the $L_{d}$ 's for each subdivided cell (see Fig. 2) in the waveguide.

For a weakly guiding waveguide, when the guide is longitudinally invariant, the circular symmetric field solutions of the scalar wave equation can be separated for the transverse and longitudinal $\{\exp (i \beta z)\}$ parts; this corresponds to the $\mathrm{LP}_{\mathrm{O} m}$ (linearly polarized) modes. Furthermore, if one of these modes is launched with a given power, that modal power will remain unchanged along the guide. However, the waveguide core (index profile) considered in this paper varies with the vertical propagating direction $z$ and radius, where the LP modes are no longer independent solutions of the corresponding scalar wave equation, i.e., modal powers do not remain invariant. Therefore, the mode profile in a nonuniform cylindrical waveguide is quite complicated. The propagation constant $\beta$ depends on $z$ [12], i.e., guided mode may exist at one end but under cutoff at the other end. One of the ways to characterize the field evolution along these nonuniform guides is by means of the coupled-mode equations [12]. This method requires local expansion of the field in terms of a complete set of modes. To construct the local mode fields, we approximate the waveguide by a series of uniform cylindrical sections as described in Fig. 2.

The refractive index profile is independent of $z$ within each section and is defined at the center, $z=z_{c}$, of each section where it coincides with the profile of the actual nonuniform waveguide, i.e., $n=n\left(r, \phi, z_{c}\right)$. We approximate the fields within the finite section by the modal fields of an infinitely long waveguide with $n\left(r, \phi, z_{c}\right)$. This approximation can be very accurate provided that the length of the section $\delta z$ is small compared to the largest length scale for the fields, as discussed below. The electric field of the $j$ th local mode has a separable form

$$
\Psi_{j}=\psi_{j}\left[r, \phi, \beta_{j}\left(z_{c}\right)\right] \exp \left[i \beta_{j} z_{c}(z)\right]
$$

within each section, where $r$ and $\phi$ are coordinates in the waveguide cross section. The $\psi_{j}$ 's are solutions of the vector eigenvalue equation, and is determined with the use of multilayer approximation [18]. Although the field expressed by (7) varies as the $\Psi$ profile travels from one section to another section, the power of a local mode must be conserved along the nonuniform waveguide structure. This requirement is automatically satisfied if we use the orthonormal forms of $\hat{\Psi}_{j}=\Psi_{j} / \sqrt{N_{j}}$, where $N_{j}=\left\|\hat{\Psi}_{j}\right\|$ for fields in each section, i.e., replacing $\psi_{j}$ by $\hat{\psi}_{j}$ in (7). As long as the local mode propagates, its phase increases across each section by the product of $\beta_{j}\left(z_{c}\right)$ and the section of length $\delta z$. Consequently, the phase at an arbitrary position along the nonuniform waveguide is a sum of such products. However, the slow variation means that the propagation constant varies only slightly from one section to another. Hence, we can accurately approximate the phase sum by $\beta_{j}(z)$ in $\hat{\psi}_{j}$. To summarize, the local mode fields at position $z$ are given by

$$
\Psi_{j}=\psi_{j}\left[r, \phi, \beta\left(z_{c}\right)\right] \exp \left[i \int_{0}^{z} \beta_{j} z_{c}(z) d z\right] .
$$

Transverse mode of the circular waveguide can be expressed in term of $\mathrm{LP}_{l_{p}}$ mode where $l$ and $p$ stand for the azimuthal and radial orders of the mode. In order to consider the nonuniform permittivity distribution in each section, we adopt the multilayer approximation to replace the actual graded-index profile with a multistep profile for analyzing the guiding mode in the waveguide. An error $\delta n$ thus is deliberately accepted, the effect of which is minimized when the average of $\delta n$ is zero in every layer. When the core profile is approximated with $N$ layers, there are $N+1$ discontinuities at $r_{0}, r_{1}, r_{2}, \cdots r_{N}$. The Helmholtz equation can be expressed by the standard Bessel functions $J_{l}, Y_{l}$ and the modified Hankel functions $K_{l}, I_{l}$ of order $l$, and the electric field is expressed as shown in (9) at the bottom of the page where $r_{m}=m \Delta r$ and $\Delta r=b / M$ so that $r_{M}=b$ and $r_{2 M}=2 b$. The propagation constant is defined as

$$
\left\{\begin{array}{l}
\kappa^{2}\left(r_{m}\right)=n^{2}\left(r_{m}\right) k_{0}^{2}-\beta^{2} \\
\gamma^{2}\left(r_{m}\right)=\beta^{2}-n^{2}\left(r_{m}\right) k_{0}^{2}
\end{array}\right.
$$

For a guided mode to occur, the propagation constant requires to meet the following criterion:

$$
n_{\min } k_{0} \leq \beta \leq n_{\max } k_{0} .
$$

Guided mode can be evaluated by considering the continuity of $\psi$ and its corresponding derivative $\partial \psi / \partial r$ in (9). In other words, the propagation constant $\beta$ of the guided mode $\mathrm{LP}_{l m}$ can be solved directly by using (9) and the derivative of this system. Note that there is no guiding mode if no solution is available for the above set of equations. Once we obtain the guided mode propagation constant, the electric field profile can be determined directly. The constant $A_{i}$ and $B_{i}$ can be found by taking $A$ to be any arbitrary constant.

For a given refractive index profile, $n(r, z)$, the propagation constant $\beta$ is calculated, where the corresponding confinement factor or fraction of power in the core is given as

$$
\eta=\frac{\int_{A_{\text {corc }}} \psi^{2}(r, z) d A}{\int_{A_{\infty}} \psi^{2}(r, z) d A}
$$

and $A_{\text {core }}$ is the area of QW layers under the mask.

$$
\psi=\exp (j \ell \phi) \exp (j \beta z) \begin{cases}A_{1} J_{\ell}\left(\kappa_{1} r\right) & 0 \leq r<r_{1} \\ A_{2} J_{\ell}\left(\kappa_{2} r\right)+B_{2} Y_{\ell}\left(\kappa_{2} r\right) & r_{1} \leq r<r_{2} \\ \vdots & \\ A_{m} J_{\ell}\left(\kappa_{m} r\right)+B_{m} Y_{\ell}\left(\kappa_{m} r\right) & r_{m-1} \leq r<r_{m} \\ A_{m+1} K_{\ell}\left(\gamma_{m+1} r\right)+B_{m+1} I_{\ell}\left(\gamma_{m+1} r\right) & r_{m} \leq r<r_{m+1} \\ \vdots & \\ A_{2 M} K_{\ell}\left(\gamma_{2 M} r\right)+B_{2 M} I_{\ell}\left(\gamma_{2 M} r\right) & r_{2 M-1} \leq r<r_{2 M} \\ A_{2 M+1} K_{\ell}\left(\gamma_{2 M+1} r\right) & r>2 b\end{cases}
$$




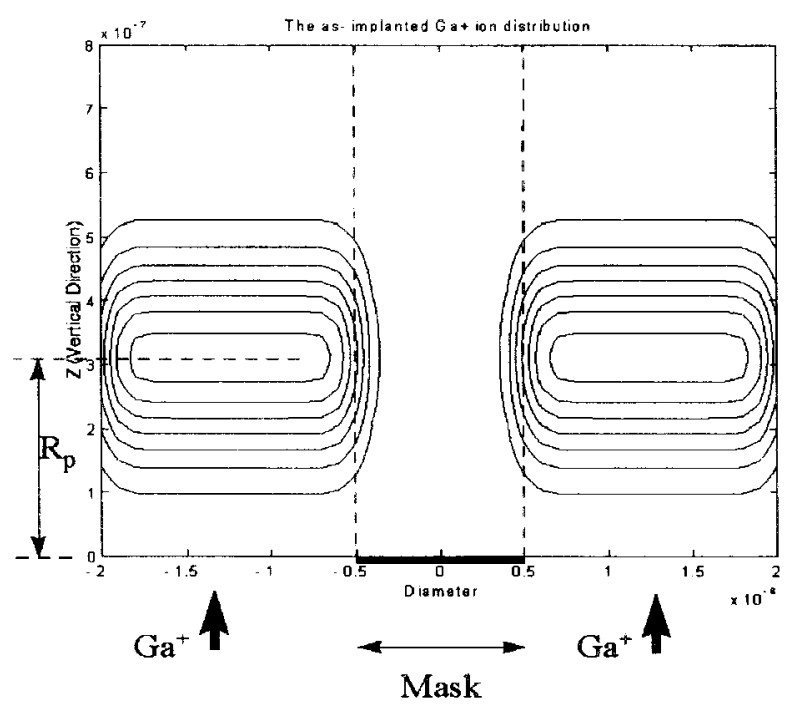

Fig. 3. The as-impurity profile (Implantation energy $=800 \mathrm{keV}$, mask radius $=0.5 \mu \mathrm{m}$ ).

\section{Result AND Discussion}

The properties of IID defined vertical cavity QW waveguide are investigated based on the model developed here. The circular waveguide structure to be analyzed here consists of a region of diameter $2 b=4 \mu \mathrm{m}$ (including a core of $2 a$ diameter and cladder) and length of cavity $H=0.8 \mu \mathrm{m}$ (unless otherwise indicated) that consists of $\mathrm{AlGaAs} / \mathrm{GaAs}$ MQW layers with thickness $100 \AA / 100 \AA$. The effect of IID to be studied includes variations of ion implantation energy $(400,600,800$ and $1000 \mathrm{keV})$, operating wavelength $(\lambda=$ $0.85,0.90,1.0$ and $1.5 \mu \mathrm{m})$, mask radius $(a=0.5,1.0$ and $1.5 \mu \mathrm{m})$ and implantation doses $\left(N_{0}=1 \times 10^{13}\right.$ and $1 \times 10^{14}$ $\mathrm{cm}^{-2}$ ); all carried out at room temperature. It is essential that all the other physical parameter and dimensions must be kept unchanged in a controlled manner. Two grid spacings are used in the model, one for implantation and the other for refractive index profiles. For the axially symmetric system, the two grid spacing are $\left(\Delta r_{1}=0.01 \mu \mathrm{m}, \Delta z_{1}=0.001 \mu \mathrm{m}\right)$ and $\left(\Delta r_{2}=0.05 \mu \mathrm{m}, \Delta z_{2}=0.02 \mu \mathrm{m}\right)$ resulting a computation domain of $200 \times 800$ in size for the impurity profile and $40 \times 40$ for that of the refractive index profile, respectively.

\section{A. Material Properties}

Each implantation energy is associated with a specific projected range $R_{p}$, vertical standard deviation $\Delta R_{p}$, and lateral standard deviation $\Delta R_{p l}$. Making use of published data [19] and simulation [20], the as-implant distribution can be determined by (1). Fig. 3 illustrates the as-implanted $\mathrm{Ga}^{+}$ concentration profile for implantation energy of $800 \mathrm{keV}$ and mask radius of $a=0.5 \mu \mathrm{m}$. It is seen that the ions have been successfully implanted to the nonmasked region and reaches a peak concentration at a depth $z=R_{p}$ while a little amount of impurities is found under the mask due to the edge effect. One should note that the ions are diffused laterally to the region under mask. The ion distribution profile during annealing is computed numerically for every time step $\Delta t$, which is taken to be $0.0005 \mathrm{~s}$. This impurity concentration profile is very

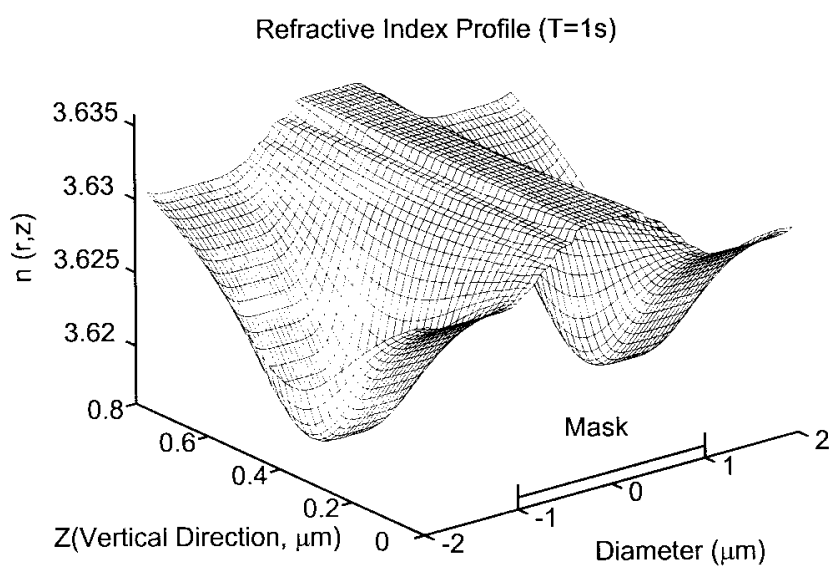

(a)

Refractive Index Profile ( $\mathrm{T}=60 \mathrm{~s})$

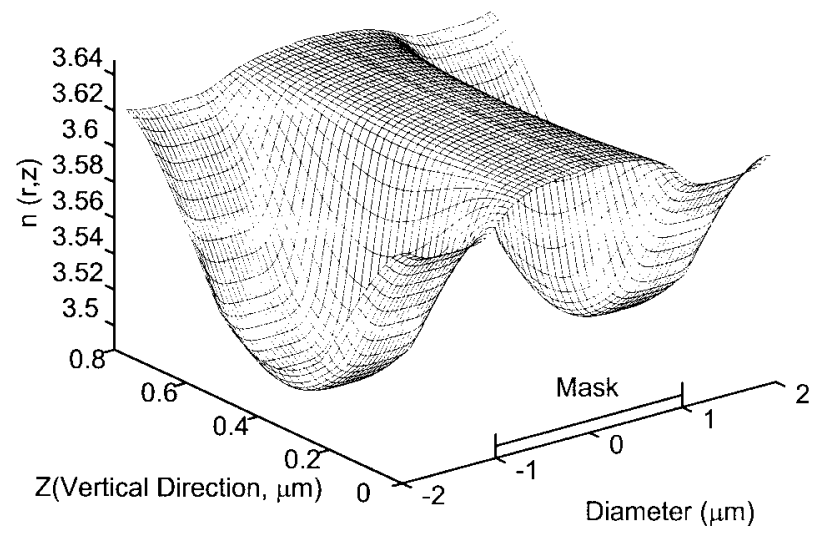

(b)

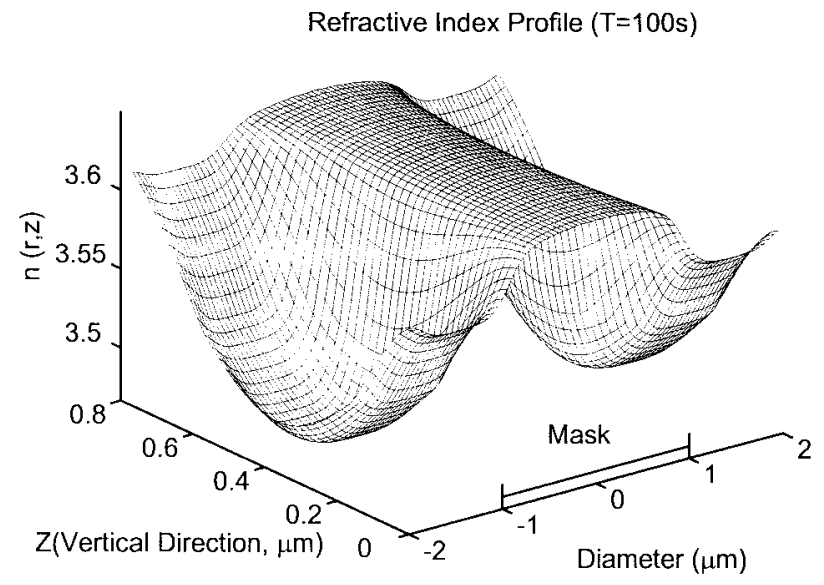

(c)

Fig. 4. Refractive index profile of the circular waveguide with mask radius $=1 \mu \mathrm{m}$, energy of implantation $=800 \mathrm{keV}$ and $\lambda=852 \mathrm{~nm}$ for various annealing time (a) $t=1 \mathrm{~s}$, (b) $t=60 \mathrm{~s}$, and (c) $t=100 \mathrm{~s}$.

essential as it affects the characteristic of the waveguiding properties in the cavity.

Due to an azimuthal invariance of the ion profile, the refractive index profile is reduced to a 2-D case. It is observed in Fig. 4 that QW's under mask has not been interdiffused and thus the corresponding refractive index remains constant at $n=3.63$. The value of the refractive index has dropped outside the mask region where interdiffusion takes place. By 
TABLE I

Modal Power Evolution Along Taper: Comparison of Simulations Using Coupled Local Mode Theory (CM) and BeAm-Propagation Method (BPM)

\begin{tabular}{c|cc|cc}
\hline \multirow{2}{*}{$z(\mu \mathrm{m})$} & \multicolumn{2}{|c|}{ LP01 } & & \multicolumn{2}{c}{ LP02 } & \\
\cline { 2 - 5 } & BPM & CM & BPM & CM \\
\hline 300 & 1 & 1 & 0 & 0 \\
350 & 0.99 & 0.996 & 0.01 & 0.01 \\
400 & 0.98 & 0.986 & 0.02 & 0.02 \\
450 & 0.905 & 0.91 & 0.08 & 0.075 \\
500 & 0.805 & 0.806 & 0.168 & 0.167 \\
550 & 0.805 & 0.804 & 0.21 & 0.206 \\
600 & 0.805 & 0.806 & 0.172 & 0.172 \\
650 & 0.82 & 0.822 & 0.12 & 0.117 \\
700 & 0.79 & 0.791 & 0.147 & 0.144 \\
750 & 0.58 & 0.581 & 0.225 & 0.223 \\
800 & 0.467 & 0.46 & 0.115 & 0.112 \\
850 & 0.344 & 0.341 & 0.096 & 0.092 \\
900 & 0.295 & 0.289 & 0.142 & 0.141 \\
\hline
\end{tabular}

carefully comparing the impurity concentration and refractive index profile (i.e., Figs. 3 and 4), it can be revealed that higher impurity concentration would lead to lower value of refractive index.

\section{B. Waveguiding Properties}

In order to provide a rough estimation of the accuracy produced by the couple mode method developed here, a nonuniform profile with constant refractive index steps is put on trial. It is obvious that accuracy increases with the number of index layers. This method is initially applied to a taper filter [21] for comparison. Table I shows the modal power (normalized by $1 / \sqrt{N_{j}}$ ) and its evolution along the taper for the first two $\mathrm{LP}_{0 m}$ modes. Results demonstrate an excellent agreement for the couple mode method, with errors less than $5 \%$, with the Beam Propagation Method. In this paper, the refractive index profile along the radial direction is divided approximately into twenty index regions, i.e., $\delta R=0.2 \mu \mathrm{m}$. The properties of the propagating wave at various vertical depth positions, $z$, are then studied by using the coupled-mode equation. To attain an acceptable accuracy, thickness of the longitudinal layers is taken as $\delta z=0.04 \mu \mathrm{m}$.

1) Guiding Modes: Waveguiding properties of the vertical type of cavity have been intensively studied recently [22]-[27]. The graph in Fig. 5 shows the 3-D mode profile and contour plot of normalized intensities for the fundamental $\mathrm{LP}_{01}$ mode along the propagation vertical direction. The mask radius of the illustrated structure is $a=1 \mu \mathrm{m}$ and the ion-implantation is carried out at $600 \mathrm{keV}$. It should be noted that, [see Fig. 5(a)], the intensity of the guided wave is varying along the propagation direction. The intensity of the field increases initially and attains its maximum value as $z$ approximately comes to a point corresponding to the projected range and which most of the light can be confined there. The amplitude of the guided wave starts to decrease as the wave goes down to the bottom $(z \approx 0.8 \mu \mathrm{m})$ of the waveguide. This shows that the wave is best guided at the depth of projected range. The
LP 01 mode along vertical direction

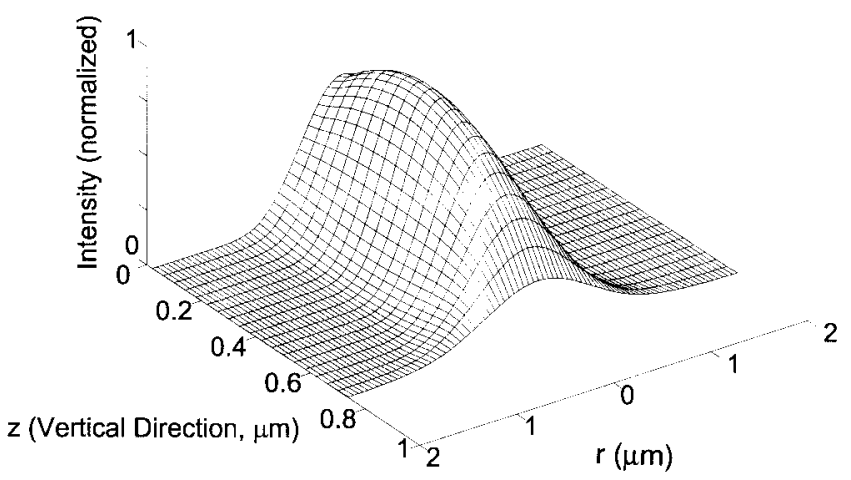

(a)

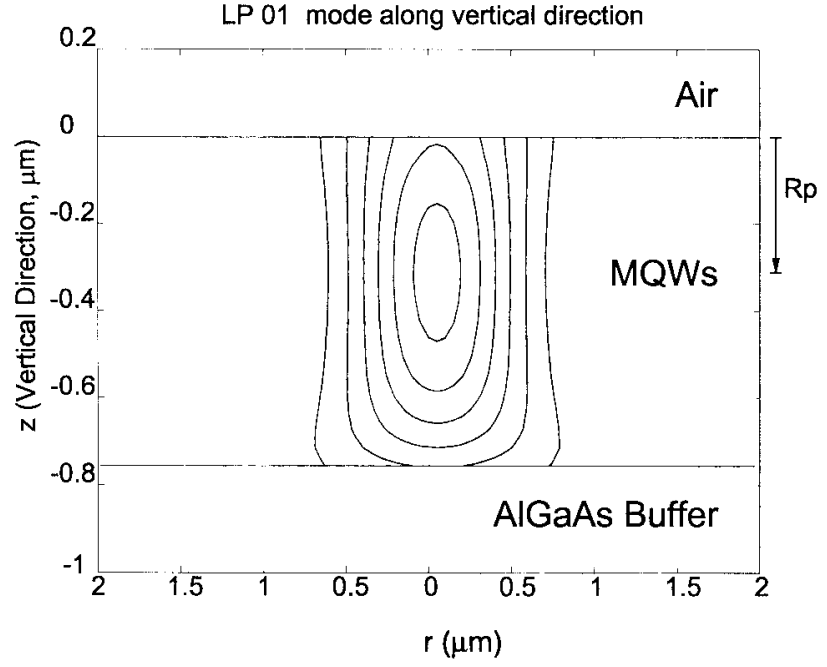

(b)

Fig. 5. Mode intensity profile for waveguide with implant energy $=600$ $\mathrm{keV}$, mask radius $=1 \mu \mathrm{m}$; annealed for $91 \mathrm{~s}$ and $\lambda=852 \mathrm{~nm}$. (a) Three-dimensional profile and (b) contour plot.

reason is that when guided wave travels to a depth near $R_{p}$, concentration of the IID impurities will be the highest. This implies that the quantum-wells nearby will be intermixed to the largest extent, which in turn leads to a bigger refractive index step between the mask and implanted regions. As depicted in the typical refractive index profile shown in Fig. 6, the spatially varied refractive index difference increases with depth and peaks at the projected range $R_{p}$. In other words, light is more and more confined in the mask region as it is traveling from $z=0$ to $R_{p}$ and then starts to be less confined as the wave travels onwards.

Furthermore, the guided mode may not be maintained throughout the entire waveguide cavity. As seen in Fig. 5(a), the wave starts to fade gradually when the guided wave travels beyond $R_{p}$. Consequently, the guide mode is under cutoff before arriving at the other end $(z=0.8 \mu \mathrm{m})$ of the cavity. This is due to the ion-implantation energy, for lower energy, ions are not capable to penetrate deep into the lower cavity quantum-wells and therefore no interdiffusion can take place in the lower part of the cavity. This implies that the refractive index difference is so small that no guided mode is supported 


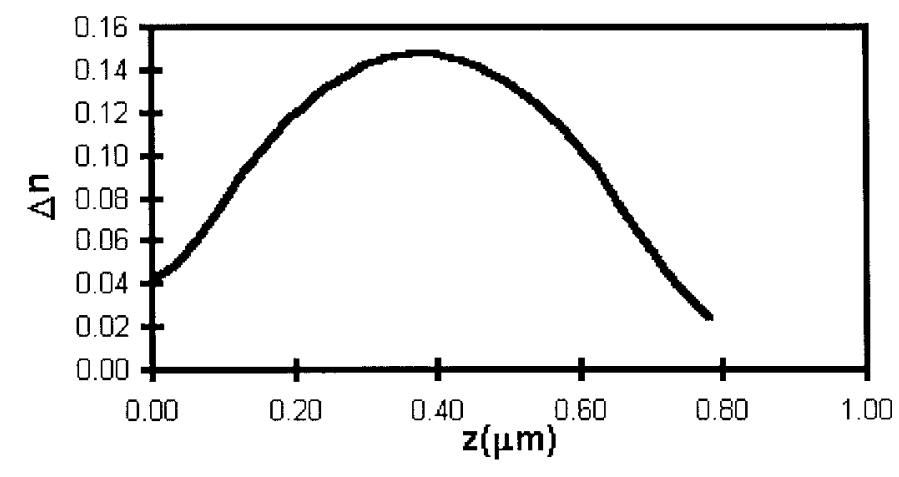

Fig. 6. Section averaged refractive index difference $\left(\Delta n=n_{\text {core }}-n_{\text {clad }}\right)$ at operating wavelength $852 \mathrm{~nm}$ and waveguide defined by implantation energy $=1000 \mathrm{keV}$, mask radius $=1 \mu \mathrm{m}$ and annealing Time $=91 \mathrm{~s}$.

there. Hence, it should be noted that the depth in which guided mode can be maintained, $l_{\text {guide, }}$ is greatly affected by $R_{p}$ and $\Delta R_{p}$, which in fact is determined by the implantation energy chosen.

The modal properties is a very important aspect of the vertical cavity waveguiding, it has been studied by several authors [22]-[27]. In addition to the $\mathrm{LP}_{01}$ mode intensity profile discussed above, other higher order modes are also depicted in Fig. 7. The normalized electric field of the modal profile are shown at a fixed propagation position $z=0.2 \mu \mathrm{m}$, they follow prediction from the vibrational mode theory accordingly [28]. The $\mathrm{LP}_{01}$ mode is the fundamental mode, being symmetrical about the longitudinal axis and has the highest strength at the centre of the cavity, it agrees well with experimental results [22], [24]. The $\mathrm{LP}_{02}$ mode is in principle similar to $\mathrm{TE}_{02}$ mode with ring pattern, i.e., lowest intensity at the centre of cavity and surrounded by a ring of lower intensity. It agrees well with experimental data [23] except there exists some discrepancies at the outer edge of the cavity, this may due to the graded refractive index produced by the IID in our model. Other even higher order modes, $\mathrm{LP}_{21}, \mathrm{LP}_{11}, \mathrm{LP}_{22}$, and $\mathrm{LP}_{12}$, are also shown for the sake of completeness. They generally are in agreement to the general theory and vertical cavity measurements [22], [24]. Some are asymmetrical about the longitudinal axis, the higher the mode order, the more complicated the nodal line is.

2) Ion Implantation: The consideration of ion implantation energy is a very crucial subject and should be investigated carefully. Ion implantation energy determines the range of the impurities penetrated, thus determines the guiding capability at various vertical positions. The depth of the penetration of impurities is best described by the projected range $R_{p}$ of the implantation, which specifies the depth where impurities are of highest concentration. The effect of $R_{p}$ on the refractive index is shown in Fig. 6 that the profile would attain the highest refractive index difference between the masked and nonmasked regions at $R_{p}$. As a result, light is best confined at a region around $R_{p}$, implying that most of the power of EM wave can be gathered there. Fig. 8 presents the variation of power confined in the masked region along the cavity with different implantation energies. An energy of $400 \mathrm{keV}$ corresponds to a $R_{p}$ of $0.15 \mu \mathrm{m}$, which in turn is the point having the peak for the optical confinement ratio $\eta$ for the line $400 \mathrm{keV}$ in Fig. 8. Similar phenomenon can be observed for cases with $600 \mathrm{keV}, 800 \mathrm{keV}$ and $1000 \mathrm{keV}$. Therefore, $\eta_{\max }$ is located at the projected range for all cases of implantation, due to a higher degree of intermixing. However, different implantation energy leads to different value of $R_{p}$. It is known that ions with higher energy can penetrate deeper during implantation. As illustrated in Fig. 8, the projected range $R_{p}$ increases from 0.152 to $0.395 \mu \mathrm{m}$ when implantation energy increases from $400 \mathrm{keV}$ to $1000 \mathrm{keV}$. Therefore, with higher implantation energy, guided mode can be maintained for a longer (deeper) cavity distance.

The effect of $R_{p}$ alone is not sufficient to characterize the guiding mode characteristics. It is obvious that the implanted impurity can spread over the cavity and it has shown to be a function of $z$ (depth) and $r$ (lateral) and assumed to be of error function shape. Such spreading effect is desired in our model where guided mode can therefore be maintained in an easier way if the degree of intermixing is sufficient enough along the whole cavity. The degree of spreading effect can be specified with the parameter $\Delta R_{p}$. It is essential to note that $\Delta R_{p}$, similar to $R_{p}$, is found to be increasing with implantation energy. Therefore, intermixing can be attained in deeper QW's if high-energy implantation is employed. The guided mode can extend to a deeper (larger $z$ value) position when higher implantation energy is used. For energy of 400 $\mathrm{keV}$, the guided mode can only maintain until $0.45 \mu \mathrm{m}$ while more than $0.7 \mu \mathrm{m}$ for that of $1000 \mathrm{keV}$ (increased by more that $60 \%$ ). The length of the channel (guided mode) can thus be increased with higher implantation energy. Therefore, higher implantation energy is preferred if thicker vertical cavity is required in design. However, higher implantation energy may lead to damage of the waveguide. It should be carried out carefully if high-energy implantation is employed. Although spreading effect is advantageous, it has drawbacks to a certain extent. If $\Delta R_{p}$ is too large, the spreading effect is too great, the concentration profile of impurities would become broadened along the direction of implantation. Thus, the ion concentration will decrease at nearby $R_{p}$, which in turn leads to a lower degree of intermixing and thus a smaller refractive index step. However, this effect is not so significant in general and may be neglected. The dosage of implanted ions is considered as another parameter for the ion-implantation. Samples of different ion concentrations, $10^{13} \mathrm{~cm}^{-2}$ and $10^{14} \mathrm{~cm}^{-2}$, are chosen to study the influence of the abundance of impurity to our model. A model with $600 \mathrm{keV}$ ion-implantation, mask radius of $1 \mu \mathrm{m}$ and annealed for $51 \mathrm{~s}$, is illustrated and is intended to operate at a wavelength of $852 \mathrm{~nm}$. It is shown in Fig. 9 that there is an extended guided mode length in the cavity for that of the $10^{14} \mathrm{~cm}^{-2}$ case, which implies that with larger ion concentration, the degree of intermixing is increased in such a way that the refractive index difference is large enough to encourage higher fraction of the power to be confined in the masked region. Moreover, such phenomenon is shown to agree with our theoretical assumption and thus a provision of accurate result.

3) Annealing: Apart from the effect of ion implantation, the process of annealing also plays a major role in our 
LP01 mode $(z=0.2 \mu \mathrm{m})$

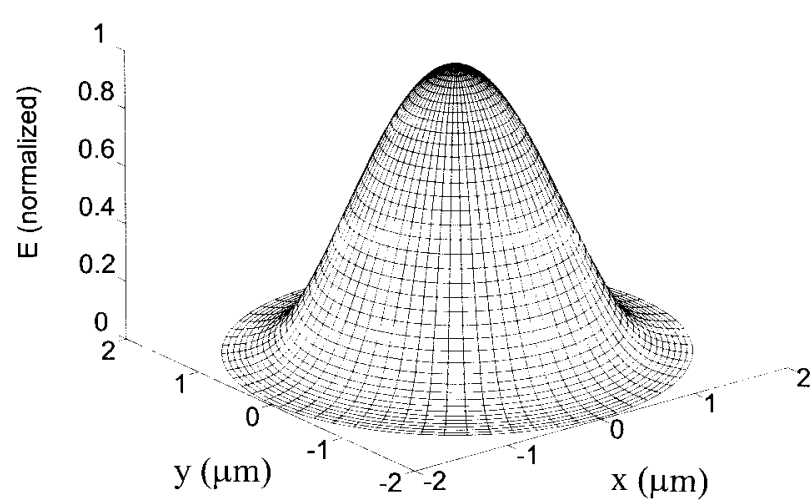

(a)

LP12 mode $(z=0.2 \mu \mathrm{m})$

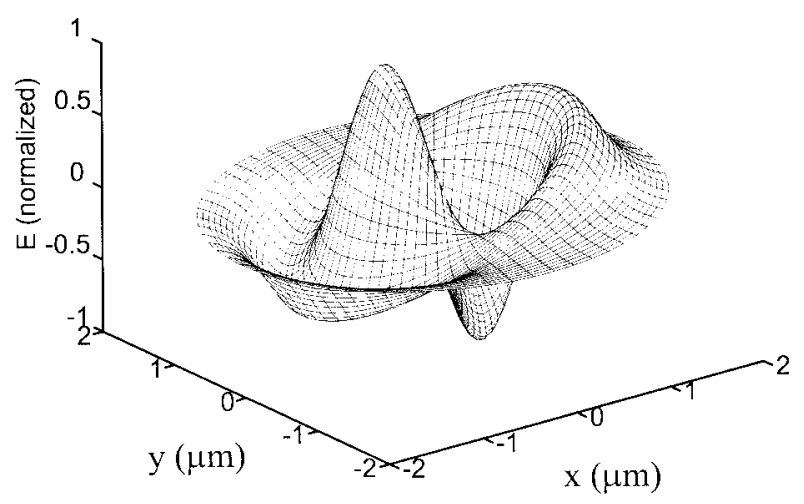

(c)

LP21 mode $(z=0.2 \mu \mathrm{m})$

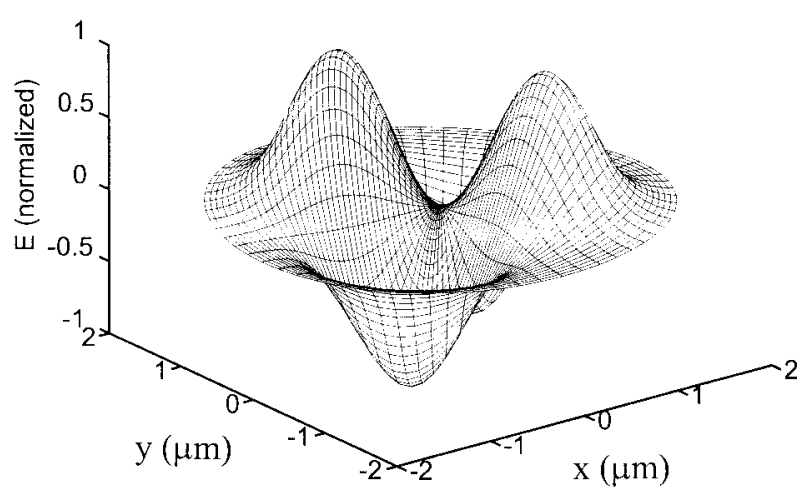

(e)
LP11 mode $(z=0.2 \mu \mathrm{m})$

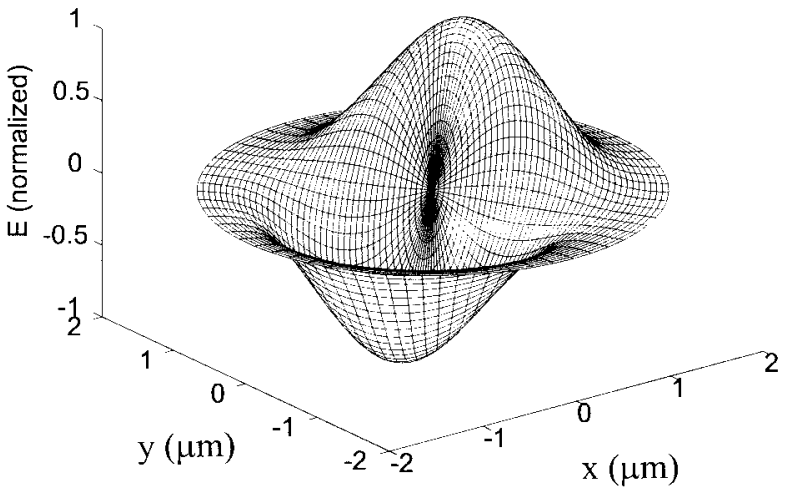

(b)

LP02 mode $(z=0.2 \mu \mathrm{m})$

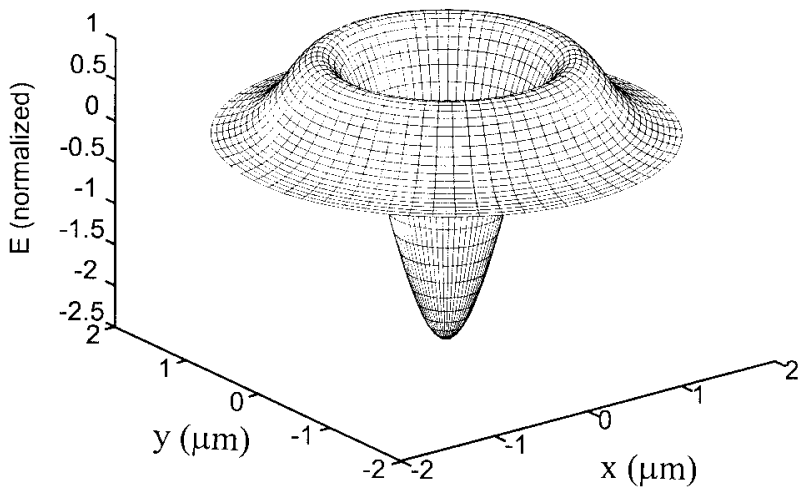

(d)

LP22 mode $(z=0.2 \mu \mathrm{m})$

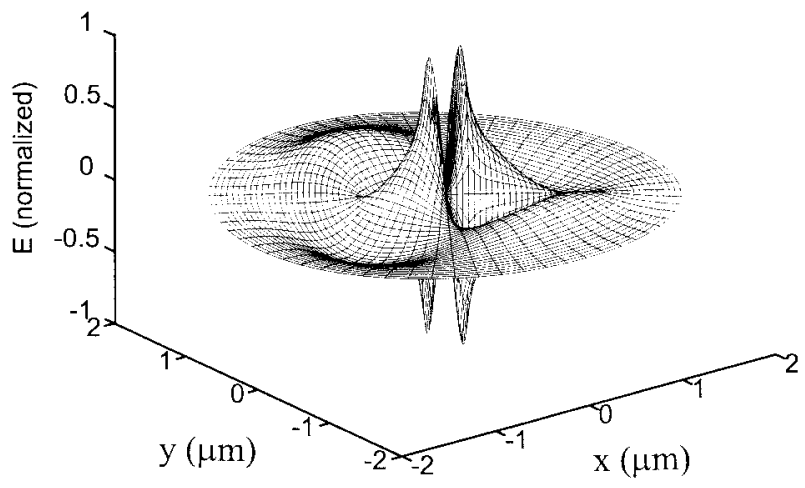

(f)

Fig. 7. Higher order linear polarized mode profiles: (a) $\mathrm{LP}_{01}$ mode, (b) $\mathrm{LP}_{11}$ mode, (c) $\mathrm{LP}_{12}$ mode, (d) $\mathrm{LP}_{02}$ mode, (e) $\mathrm{LP}_{21}$ mode, and (f) $\mathrm{LP} 22$ mode. IID parameters: ion-implantation energy $=800 \mathrm{keV}$, mask diameter $=3 \mu \mathrm{m}$, annealing time $=40 \mathrm{~s}, \lambda=892 \mathrm{~nm}$.

waveguide model. The effect of annealing enhances diffusion of the impurity ions and provides a remarkable modification in the refractive index profile. In Fig. 10, the influence of annealing on waveguide structure is demonstrated. The structure annealed is implanted with $600 \mathrm{keV} \mathrm{Ga}$ ions and a mask of radius $a=1 \mu \mathrm{m}$ is placed on it to provide the refractive index step. The changes of the performance of guided mode with time, from 1 to $91 \mathrm{~s}$, during high temperature annealing are investigated. The essence of annealing is clearly expressed. In the as-implanted index profile (annealing time $t=1$ s), the refractive index difference between the masked and nonmasked region is not great enough to reach satisfactory guiding result. Only less that $80 \%$ of the power can be gathered in the "core" region and the depth of guided mode region $l_{\text {guide }}$ is not so long. After annealing for ten seconds, however, there is a noticeable modification in the guiding properties of the 


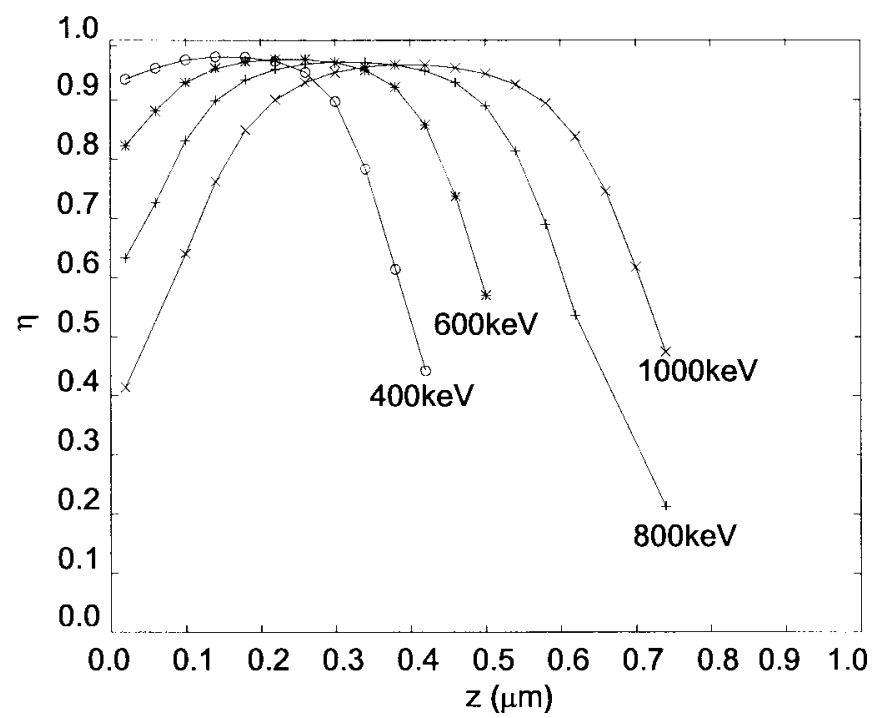

Fig. 8. Characteristic of optical confinement $(\eta)$ properties at $\lambda=850 \mathrm{~nm}$ as a function of propagation direction ( $z$-axis) for several cases of implantation energy where the waveguide is defined by mask radius $=1 \mu \mathrm{m}$ and duration of annealing $=51 \mathrm{~s}$.

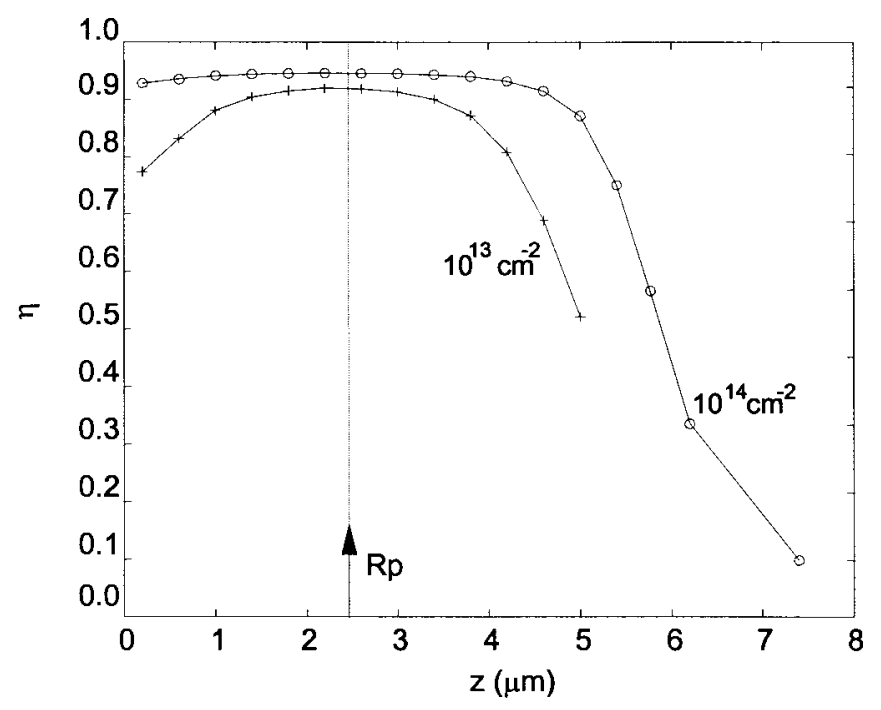

Fig. 9. Characteristic of optical confinement $(\eta)$ properties at $\lambda=850 \mathrm{~nm}$ as function of propagation ( $z$-axis) for two cases of ion dosage. The waveguide is defined by implantation energy $=600 \mathrm{keV}$, mask radius $=1 \mu \mathrm{m}$, and annealing time $=51 \mathrm{~s}$.

structure. The optical confinement factor has increased to over $90 \%$ and the guided mode can propagate deeper than before. Such drastic enhancement of the guided mode properties has proven the indispensable effect of the QW intermixing in our structure. On the other hand, the enhancement of the waveguide quality will be less remarkable than before as annealing prolongs further. The fraction of power confined in the masked region becomes constant for small depth $z$ (less than $0.4 \mu \mathrm{m}$ ) and the curves in Fig. 10 become closer and closer together as annealing carries on. Hence, the rapid thermal annealing of QW's can really serve as an efficient mean in fabricating a waveguide device defined by IID.

4) Operating Wavelength: When studying the relationship between operating wavelength and the mode properties, it is discovered that different wavelength of light can have

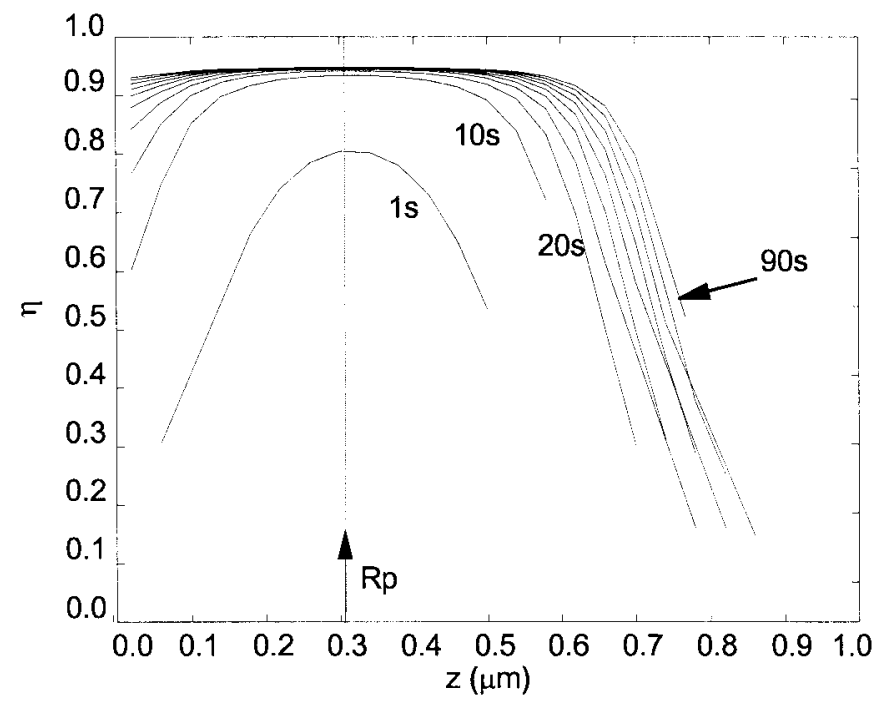

Fig. 10. Characteristic of optical confinement $(\eta)$ properties at $\lambda=850 \mathrm{~nm}$ as function of propagation ( $z$-axis) for different duration of annealing. The waveguide is defined by implant energy $=800 \mathrm{keV}$ and mask radius $=1 \mu \mathrm{m}$.

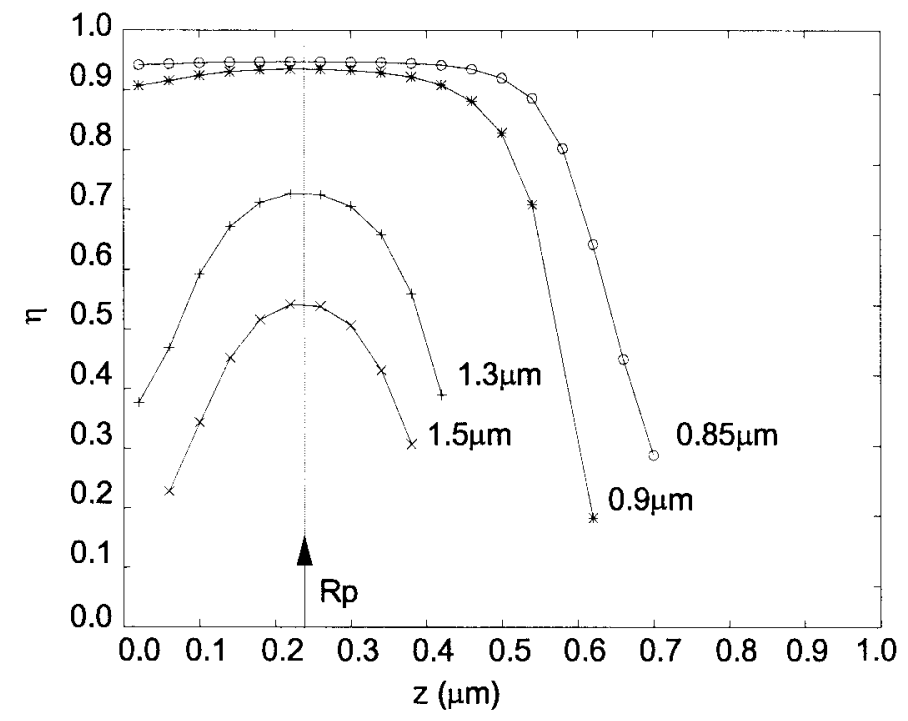

Fig. 11. Characteristic of optical confinement $(\eta)$ properties as function of propagation ( $z$-axis) operated at different wavelength. The waveguide is defined by implantation energy $=600 \mathrm{keV}$; duration of annealing $=91 \mathrm{~s}$; mask radius $=1 \mu \mathrm{m}$.

different guiding properties. As an example is illustrated in Fig. 11, fundamental $\mathrm{LP}_{01}$ mode arises more likely for shorter wavelength $(0.852$ and $0.95 \mu \mathrm{m})$. The model has been annealed for $91 \mathrm{~s}$ and the range of ion projected is $0.231 \mu \mathrm{m}$. The four associated propagation constant $\beta$ derived from the different operating wavelength are different from each other. In case of longer wavelength operation (1.3 and $1.5 \mu \mathrm{m})$, guided mode is less likely to be seen and only with a small range along the $z$-direction. Moreover, the corresponding fraction of light confined is comparatively small (less than $70 \%$ ). On the contrary, the guided mode of shorter wavelength is managed to propagate to a deeper QW region. There is more than $90 \%$ of the fraction of power confined at a range of depth's less than $0.5 \mu \mathrm{m}$. This is because the refractive index profile is wavelength dependent and in such a way that the longer the 


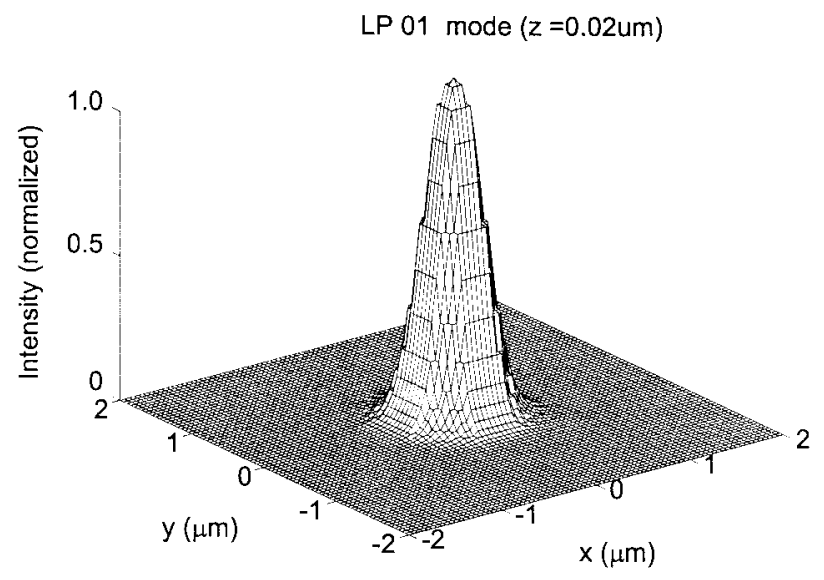

(a)

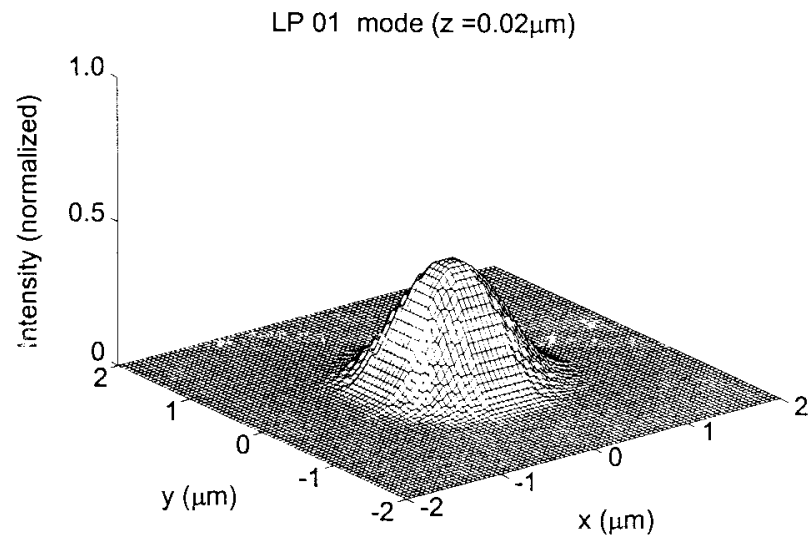

(b)

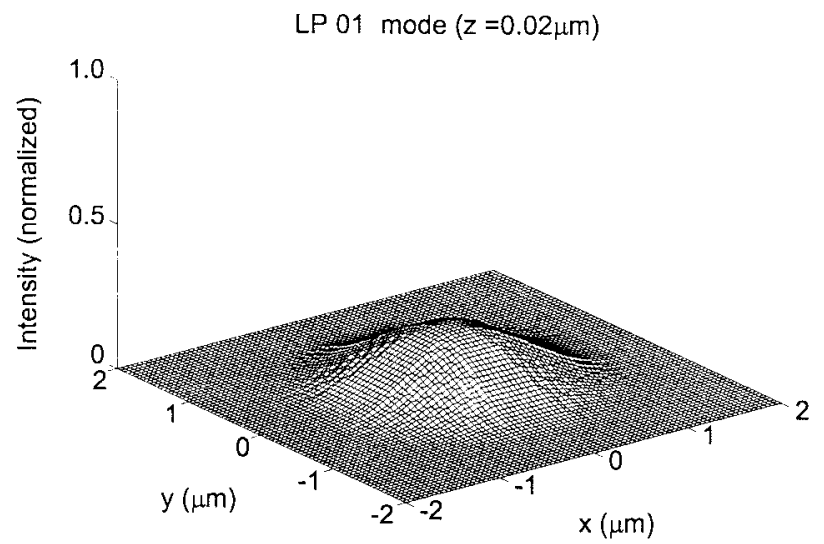

(c)

Fig. 12. Optical mode intensity profile for different mask radius of (a) 0.5 $\mu \mathrm{m}$, (b) $1 \mu \mathrm{m}$, and (c) $1.5 \mu \mathrm{m}$, respectively (Implantation energy $=400 \mathrm{keV}$, duration of annealing $=51 \mathrm{~s}, \lambda=852 \mathrm{~nm}$ ).

operating wavelength, the smaller the index difference between masked and nonmasked region. As a result it is less probable to attain guided mode operation for longer wavelength. In short, the proposed structure is suitable for operation at wavelength 0.85 or $0.9 \mu \mathrm{m}$. For the cases of $\lambda=1.3$ or $1.5 \mu \mathrm{m}$, the guiding behavior is not satisfactory and is not suggested here.

5) Structural Dimension: Since the thickness of each QW layer is constant, the thickness of the cavity can be adjusted by controlling the number of QW layers included. In practical

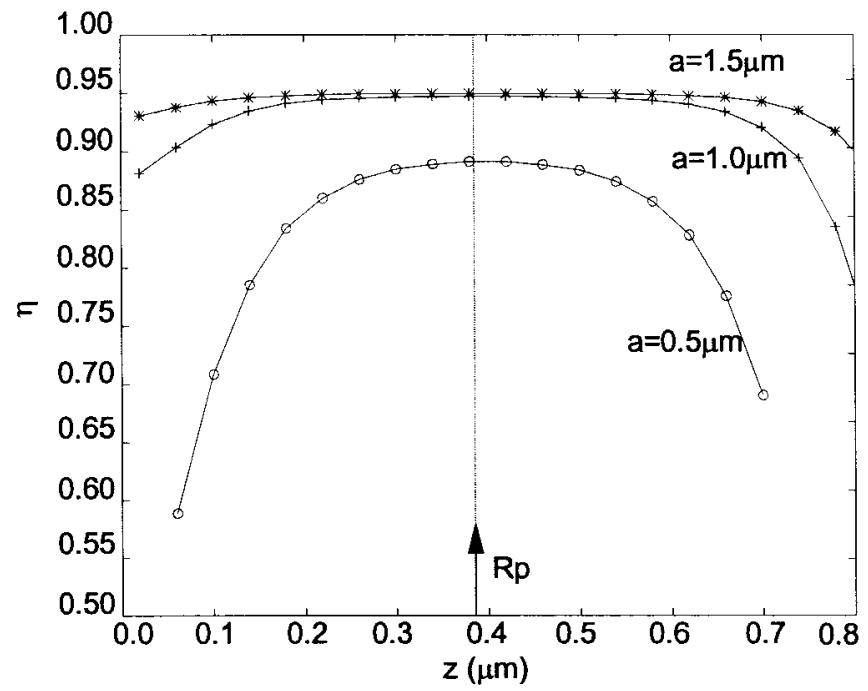

Fig. 13. Characteristic of optical confinement $(\eta)$ properties at $\lambda=850 \mathrm{~nm}$ as function of propagation ( $z$-axis) for different cases of mask radius. The waveguide is defined by implantation energy $=1000 \mathrm{keV}$ and duration of annealing $=71 \mathrm{~s}$.

consideration [29], a limit of 40 QW layers, i.e., $0.8 \mu \mathrm{m}$ in thickness is adopted. As studied before, the distance of which the guided mode can be maintained is mainly subject to the implantation of impurity ions. Thus, in designing the vertical cavity defined by IID technique, the number of QW layers adopted should match with the energy of ion-implantation. As the implantation energy increases, the depth of ion diffusion also increases, thus more layers of QW can be used.

As far as the dimension of the model is of interest, the size of mask governs the impurity distribution and thus also deserves attention. A structure implanted with 1000-keV ions and annealing for 71 seconds is presented (Figs. 12 and 13). Results show that the behavior of guiding mode of the structure with mask radius $a=0.5 \mu \mathrm{m}$ is far distinct from that with $a=1.0$ and $1.5 \mu \mathrm{m}$. As seen in Fig. 12, the intensity mode profile is greatest for structure with mask radius $a=0.5 \mu \mathrm{m}$, when compared with cases of $a=1.0$ and $1.5 \mu \mathrm{m}$. Thus, intensity increases as mask radius decreases. On the other hand, in Fig. 13, it is shown that as the mask radius decreases from 1.5 to $0.5 \mu \mathrm{m}$, the distortion of $\eta$ increases and the refractive index under mask is no longer uniform, implying a weaker optical lateral confinement. This is solely due to the fact that the impurities implanted will inevitably diffuse to the region under mask. The phenomena carries on as the cavity is continuously annealed because annealing will induce the diffusion of ion into the masked region. The distortion effect is owing to the lateral projected standard deviation $\Delta R_{p l}$ of corresponding ion-implantation. It is also necessary to note that $\Delta R_{p l}$ is independent on the mask radius, but rather on the implantion energy. As indicated by Fig. 13, the influence of $\Delta R_{p l}$ would be relatively greater for narrow mask cases. Consequently, the peak value of $\eta$ decreases as the mask radius decreases. At the same time, when comparing Fig. 12 to Fig. 13, a mismatch between light intensity and $\eta$ is observed. Thus, the structure with the smallest mask radius $(a=0.5 \mu \mathrm{m})$ is the one having the weakest lateral confinement due to the distortion effect as mentioned above. 


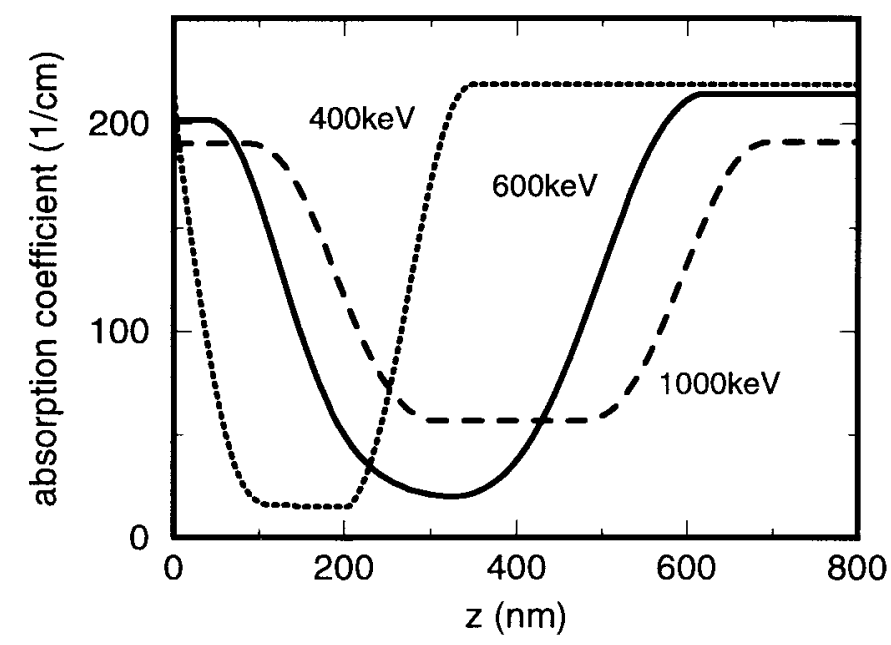

Fig. 14. Absorption coefficient with different ion-implantation energies.

6) Loss: Loss is an essential issue in waveguide design. The loss of the waveguide considered here mainly consists of two parts, namely scattering loss and material loss. At the top and bottom ends of the waveguide, the light is scattered laterally. In the waveguide proposed, consists of a core of $1 \mu \mathrm{m}$ in radius and $0.8 \mu \mathrm{m}$ in propagation length, the scattering loss is necessary to be considered [30], [31]. This loss is estimated to be $3 \mathrm{~cm}^{-1}$ using the mode matching method [32]. When light passes along the waveguide cavity, it will attenuate due to the absorption loss in the quantum-well material. Here, the loss due to three ion implantation energies, $400 \mathrm{keV}, 600 \mathrm{keV}$ and $1000 \mathrm{keV}$ are considered. In the centre of the masked region, quantum-well intermixing is at a minimum because the implanted ions from the nonmasked region cannot diffuse into it for the annealing temperature and time considered here; $L_{d}$ is calculated to be only about $0.5 \AA$ using experimental values of diffusion coefficient [33]. However, the loss is different in the nonmasked region.

For the loss in the nonmasked region, the diffusion coefficient of $A l$ is given by $D_{A l}=\alpha D_{\mathrm{imp}} N(r, z, t)$, and the annealing time is $91 \mathrm{~s}$, the diffusion length is calculated by $L_{d}=\left(D_{A l} t\right)^{1 / 2}$. For $\lambda=0.85 \mu \mathrm{m}$, the absorption coefficient is given as $\alpha(\omega)=\omega \varepsilon_{2}(\omega) / c n(\omega)$ where $\varepsilon_{2}(\omega)$ is the imaginary part of dielectric constant, $c$ is the speed of light in vacuum, and $n(\omega)$ is the refractive index.

The absorption coefficient is calculated using experimental data [34] along the vertical waveguide for different ionimplantation energies and is shown in Fig. 14. They are weighted and averaged along the $z$-direction and the average absorption coefficients are tabulated in Table II. In the centre of the masked region of the waveguide, the diffusion coefficient is determined to be $9.0 \times 10^{-16} \mathrm{~cm}^{2} \mathrm{~s}^{-1}$ at $\left(870{ }^{\circ} \mathrm{C}\right.$ for $91 \mathrm{~s}$ annealing time) which remains unchanged throughout the annealing process [33]. The diffusion length $L_{d}$ is calculated to be $20 \AA$.

Due to the quantum-well intermixing, absorption edge will shift to higher energy and hence lower the absorption loss. The absorption coefficient is approximately $10 \mathrm{~cm}^{-1}$ for $\lambda=0.85 \mu \mathrm{m}$ (i.e., $1.459 \mathrm{eV}$ ) [34]. The optical confinement
TABLE II

Absorption CoEFFicient (Averaged Over CAVITY LeNGTH) IN THE NONMASKED REgION

\begin{tabular}{c|c}
\hline $\begin{array}{c}\text { ion implantation } \\
\text { energy }(\mathrm{keV})\end{array}$ & $\begin{array}{c}\text { Absorption coefficient } \\
\left(\mathrm{cm}^{-1}\right)\end{array}$ \\
\hline 400 & 133 \\
\hline 600 & 97 \\
\hline 1000 & 88 \\
\hline
\end{tabular}

TABLE III

Total Loss with DifFERENT ION-IMPLANTATION ENERgIES

\begin{tabular}{ccc}
\hline $\begin{array}{c}\text { Ion-implantation energy } \\
(\mathrm{keV})\end{array}$ & Total loss $\left(\mathrm{cm}^{-1}\right)$ & Loss $(\mathrm{dB})$ \\
\hline 400 & 50 & -0.034 \\
600 & 39 & -0.027 \\
1000 & 36 & -0.025 \\
\hline
\end{tabular}

ratio is already determined to be about $\eta \approx 70 \%$, so that the total loss is given by $\alpha_{\text {total }}=\alpha_{s}+\alpha_{\text {core }} \eta+\alpha_{\text {clad }}(1-\eta)$ where $\alpha_{s}$ is the average scattering loss, $\alpha_{\text {core }}$ is the loss of centre of the masked region, $\alpha_{\text {clad }}$ is the loss in nonmasked region.

The total loss is tabulated in Table III. The loss is not too high as compared to other laser devices $\left(\cong 20-30 \mathrm{~cm}^{-1}\right)$ [35]. As the ion implantation energy increases, the total loss decreases because the quantum-well intermixing is more spread out throughout the $z$-direction (see Fig. 14), and therefore give rise to lower loss. It should be noted that if the waveguide is to be operated at $\lambda>0.85 \mu \mathrm{m}$, i.e., away from the band-edge, the loss can be much reduced.

\section{CONCLUSION}

In this paper, the behavior of AlGaAs/GaAs QW vertical cavity waveguide is analyzed in a consistent manner. The lateral optical confinement in the cavity is produced by impurity-induced disordering (IID) through ion implantation.

A 2-D coupled-mode equation is employed to evaluate the waveguiding condition of the structure. Results show that with a higher concentration of implanted ions, quantum-well interdiffusion can be enriched and in turn a better optical confinement can be achieved. Furthermore, guided mode can be maintained in the deeper region if higher implantation energy is adopted. However, it is not desired in cases of smaller diameter mask due to lateral spreading of impurities into the masked region. There is also a restriction in the operating wavelength in the guiding structure, where shorter wavelength (800-900 nm) is recommended. With annealing, an over $30 \%$ increase in the fraction of power gathered in the masked region is obtained. Moreover, the loss is not too high which is only around $40 \mathrm{~cm}^{-1}$. This gives a less than 0.1 $\mathrm{dB}$ loss for our waveguides. In summary, lateral confinement can be successfully achieved (about 70\%) if appropriate QW intermixing conditions are employed.

The intermixed multiple-QW structure accomplished in this paper is assumed to be only an ideal passive waveguide where the effect of the gain and carriers are neglected. In future works, the IID technique will be considered not only for waveguides, but also for other vertical cavity devices such as surface emitting lasers and modulators. 


\section{ACKNOWLEDGMENT}

The authors would like to acknowledge W. Y. Lam of the University of Hong Kong for his technical support.

\section{REFERENCES}

[1] K. Iga, F. Koyama, and S. Kinoshita, "Surface emitting semiconductor lasers," IEEE J. Quantum Electron., vol. 24, pp. 1845-1855, 1988.

[2] Y. Li, M. G. Brown, T. Dicarlo, I. Eliashevich, C. A. Tran, M. J. Schurman, T. Salagaj, R. F. Karlicek Jr., R. A. Stall, L. Koszi, Y. C. Lu, and H. Shen, "Fabrication of blue InGaN quantum well lightemitting diodes," in Proc. SPIE's Photon. West-Light-Emitting Diodes. Research, Manufacturing, and Applications II, Conf. 3279-02, San Jose, CA, Jan. 24-30, 1998.

[3] W. C. H. Choy, E. H. Li, and J. Micallef, "The polarization insensitive electro-absorptive and refractive modulation by utilizing InGaAsP/InP diffused quantum wells," IEEE J. Quantum Electron., vol. 33, pp. 382-389, 1997

[4] G. L. Christenson, A. T. T. D. Tran, Z. H. Zhu, Y. H. Lo, M. Hong, J. P. Mannaerts, and R. Bhat, "Long-wavelength resonant vertical-cavity LED/photodetector with a 75-nm tuning range," IEEE Photon. Technol. Lett., vol. 9, pp. 725-727, 1997.

[5] K. Kasahara, "VSTEP-based smart pixels," IEEE J. Quantum Electron., vol. 29 , pp. 757-768, 1993.

[6] J. W. Scott, D. B. Young, B. J. Thibeault, M. G. Peters, and L. A. Coldren, "Design of index-guided vertical-cavity lasers for low temperature-sensitivity, sub-milliamp thresholds, and single-mode operation," IEEE J. Quantum Electron., vol. 1, pp. 638-648, 1995.

[7] S. Tang, R. T. Chen, L. Garrett, D. Gerold, and M. M. Li, "Design limitations of highly parallel free-space optical interconnects based on arrays of vertical cavity surface-emitting laser diodes, microlenses, and photodetectors," J. Lightwave Technol., vol. 12, pp. 1971-1975, 1994.

[8] T. Honda, F. Koyama, and K. Iga, "Design consideration of GaN-based surface emitting lasers," Mat. Res. Soc. Symp. Proc., 1997, vol. 449, pp. $1151-1159$.

[9] P. D. Floyd, B. J. Thibeault, J. Ko, D. B. Young, L. A. Coldren, and J. L. Merz, "Vertical cavity lasers with Zn impurity-induced disordering (IID) defined active regions," in Proc. IEEE Laser Electro-Optics Soc. 1996 Annu. Meeting, 1996, pp. 207-208.

[10] E. H. Li, Ed., Quantum Well Mixing and Optoelectronic Device Applications. Milestone Series, WA: SPIE, Bellingham, vol. 145, 1998

[11] C. J. Chang-Hansnain, M. Orenstein, A. V. Lehmen, L. T. Florez, J. P. Harbison, and N. G. Stoffel, "Transverse mode characteristics of vertical cavity surface-emitting lasers," Appl. Phys. Lett., vol. 57, pp. 218-220, 1990.

[12] A. W. Snyder and J. D. Love, Optical Waveguide Theory. London, U.K.: Chapman and Hall, 1993, ch. 28, pp. 19 and 29.

[13] E. S. Koteles, S. Charbonneau, P. Poole, J. J. He, M. Davies, M. Dion, G. Aers, Y. Feng, I. V. Mitchell, and R. D. Goldbery, "Photonic integer using quantum well shape modulation," Phys. Canada, pp. 251-255, Sept./Oct. 1996

[14] T. Wolf, C. L. Shieh, R. Engelmann, K. Alavi, and J. Mantz, "Lateral refractive index step in GaAs/AlGaAs multiple quantum well waveguides fabricated by impurity induced disordering," Appl. Phys. Lett., vol. 55. pp. 1412-1414, 1989.

[15] S. Furuka, H. Matsumura, and H. Ishiwara, "Theoretical considerations on lateral spread of implanted ions," Japan J. Appl. Phys., vol. 11, pp. 134-142, 1972.

[16] T. E. Schlesinger and T. F. Kuech, "Determination of the interdiffusion of $\mathrm{Al}$ and $\mathrm{Ga}$ in undoped (Al, Ga)As/GaAS quantum wells," Appl. Phys. Lett., vol. 49, pp. 519-521, 1986.

[17] E. H. Li, B. L. Wesis, K. S. Chan, and J. Micallef, "Polarization dependent refractive index of an interdiffusion induced $\mathrm{AlGaAs} / \mathrm{GaAs}$ quantum well," Appl. Phys. Lett., vol. 62, pp. 550-552, 1992.

[18] C. Vassallo, Optical Waveguide Concept, United States: Elsevier, chap. $5,1991$.

[19] J. F. Gibbons, W. S. Johnson, and S. W. Mylroie, Projected Range Statistics. Dowden, PA: Hutchinson and Ross, 1975.

[20] J. F. Ziegler, SRIM-96 Software (A New Version of TRIM). NY: IBMResearch, Yorktown Heights, NY, 1996.
[21] F. Gonthier, A. Henault, S. Lacroix, R. J. Black, and J. Bures, "Modecoupling in nonuniform fibers-Comparison between coupled-mode theory and finite-difference beam-propagation method simulations," $J$. Opt. Soc. Amer. B, vol. 8, pp. 416-421, 1991.

[22] J. Y. Law and G. P. Agrawal, "Mode-partition noise in verticalcavity surface-emitting lasers," IEEE Photon. Technol. Lett., vol. 9, pp. 437-439, 1997.

[23] D. Burak and R. Binder, "Cold-cavity vectorial eigenmodes of VCSEL's," IEEE J. Quantum Electron., vol. 33, pp. 1205-1215, 1997.

[24] W. Nakwaski and R. P. Sarzala, "Analysis of transverse modes in gain-guided vertical-cavity surface-emitting lasers," in Proc. IEEE Laser Electro-Optics Soc. 1997 Annu. Meeting (LEOS'97), 1997, pp. 293-294.

[25] B. J. Koch, J. R. Leger, A. Gopinath, and Z. Wang, "Single-mode vertical cavity surface emitting laser by graded-index lens spatial filtering," Appl. Phys. Lett., vol. 70, pp. 2359-2361, 1997.

[26] A. Valle, L. Pesquera, and K. A. Shore, "Polarization behavior of birefringent multitransverse mode vertical-cavity surface-emitting lasers," IEEE Photon. Technol. Lett., vol. 9, pp. 557-559, 1997.

[27] G. Giaretta, M. Y. Li, G. S. Li, W. Yuen, and C. J. Chang-Hasnain, "A novel $4 \times 8$ single-mode independently addressable oxide-ioslated VCSEL array," IEEE Photon. Technol. Lett., vol. 9, pp. 1196-1198, 1997.

[28] M. A. Pinsky, Partial Differential Equations and Boundary-Valued Problems with Applications, 2nd Edition. New York: McGraw-Hill International, 1991, pp. 195-198.

[29] R. Michalzik and K. J. Ebeling, "Modeling and design of protonimplanted ultralow-threshold vertical-cavity laser-diodes," IEEE $J$. Quantum Electron., vol. 29, pp. 1963-1974, 1993.

[30] E. R. Hegblom, D. I. Babic, B. J. Thibeault, and L. A. Coldren, "Estimation of scattering losses in dielectrically apertured vertical cavity lasers," Appl. Phys. Lett., vol. 68, pp. 1757-1759, 1996.

[31] D. G. Deppe, T.-H. Oh, and D. L. Huffaker, "Comparison of verticalcavity surface-emitting lasers with half-wave cavity spacers confined by single-oxide or double-oxide apertures," IEEE Photon. Technol. Lett., vol. 9, pp. 875-877, 1997.

[32] S. F. Yu, "Analysis and design of VCSEL's for self-sustained pusation operation," IEEE J. Quantum Electron., vol. 34, pp. 497-505, 1998.

[33] J. D. Ralston, S. O'Brien, G. W. Wicks, and L. F. Eastman, "Roomtemperature exciton transitions in partially intermixed GaAs/AlGaAs superlattices," Appl. Phys. Lett., vol. 52, pp. 1511-1513, 1988.

[34] J. D. Ralston, W. J. Schaff, D. P. Bour, and L. F. Eastman, "Room-temperature exciton electroabsorption in partially intermixed GaAs/AlGaAs quantum well waveguides," Appl. Phys. Lett., vol. 54, pp. 534-536, 1988.

[35] M. Asada and Y. Suematsu, "The effect of loss and nonradiative recombination on the temperature dependence of threshold current in 1.5-1.6 $\mu \mathrm{m} \mathrm{GaInAsP/InP} \mathrm{lasers,"} \mathrm{IEEE} \mathrm{J.} \mathrm{Quantum} \mathrm{Electron.,} \mathrm{vol.} \mathrm{19,}$ pp. 917-923, 1983

Alex T. H. Li (M'97) received the B.S. degree in mathematical science from Hong Kong Baptist University, Hong Kong, in 1994 and the Ph.D. degree in electrical and electronic engineering from the University of Hong Kong in 1997.

His research interest is in the field of III-V semiconductor quantum-well waveguides. He is currently a Consultant of the Laser Computer Systems Limited, Hong Kong.

Chun-Chung Chan, photograph and biography not available at the time of publication.

E. Herbert Li (M'84-SM'95), photograph and biography not available at the time of publication. 